SCIENCE CHINA

Technological Sciences

- Editorial •

January 2018 Vol.61 No.1:1

https://doi.org/10.1007/s11431-017-9187-y

\title{
Science China Technological Sciences: Taking the great opportunity and embracing much brighter prospects
}

Science China Technological Sciences (SCTS) is a multidisciplinary journal that reports research work in technological sciences and engineering sciences. Since launched in 1950 the journal series of Science China have been making considerable contributions to the scientific and technological communications, thereby greatly promoting the development in science and technology, especially in China. SCTS was converted from Science China E in 1996 and SCTS published papers bilingually in Chinese and English during 1996-2013. In 2013 SCTS was divided into two individual journals: English edition SCTS and Chinese edition SCTS. Both English edition SCTS and Chinese edition SCTS share the same editorial board, but publish different papers. The English edition Science China Technological Sciences was indexed by SCI from its first issue in 1996 as one of the earliest SCI-index journals hosted in China. Its impact factor is 1.719 in the year of 2017, indicating that SCTS is currently an excellent journal in China. Aiming at becoming one of the top journals in the world in the area of technological sciences and engineering sciences, the new editorial board will do its best to continuously enhance the quality and international impact of the journal. The current editorial system will be further improved to provide more convenient and professional editorial operations in the manuscript submission and in the peer-review process. The review and printing period will be further shortened to ensure the as quickly as possible publication.

SCTS takes the Branch of Technological Sciences in Chinese Academy of Sciences as its home base and focuses on publishing original work and cutting-edge achievements in technological sciences and engineering sciences. The journal and the new editorial board will continuously improve the editorial assistance for authors and readers to capture the significance of diverse content of different subjects in technological sciences and engineering sciences. In recent years, some hot articles and frontier topics in SCTS have attracted extensive attention. For example, more than 10 Essential Science Indicators (ESI) papers were selected in 2017 from SCTS. The special topics "Network Science and Engineering" and "Time Delay, Noise on Dynamic Systems" published in 2016 have received warm responses. The journal also devotes itself to build up an outstanding communication platform for authors and readers in the global community of technological sciences and engineering sciences. In addition to the regular Articles and Reviews, the journal currently publishes Letters and News \& Views to immediately report novel discovery and breakthrough innovation in technological sciences and engineering sciences. We strongly encourage academicians, editorial members, and other excellent scholars in related subjects to recommend original scientific papers, including theirs owns', to be published in SCTS. The rapid progress in science, technology, and engineering in China provides the wonderful and historic opportunity for all the journals hosted in China. It is the right time for SCTS to grab the opportunity tightly. In the coming year, the editorial board will, by any means, promote the reputation of SCTS and bring further much brighter prospects to the journal.

On behalf of the entire new SCTS editorial board elected in 2017, I would like to thank all authors, reviewers and readers for your consistent support. My sincere thanks also extend to the former editorial board, as well as the editorial staff, for their vision, passion, and dedication. Research papers with high quality, innovation and significance in multidisciplinary engineering and technological subjects are invited from all over the world to be published in Science China Technological Sciences.

Editor-in-Chief: ZHANG TongYi

Materials Genome Institute of Shanghai University, Shanghai 200444, China 